\title{
Light Intensity Dependence of Current Density- voltage Characteristics of an Organic Solar Cell and Dominance Switching Between Shockley-read-hall and Radiative Recombination Losses
}

Shin Young Ryu

Ajou University

$\mathrm{Na}$ Young $\mathrm{Ha}$

Ajou University

Y. H. Ahn

Ajou University

Ji-Yong Park

Ajou University

Soonil Lee ( $\square$ soonil@ajou.ac.kr)

Ajou University

\section{Research Article}

Keywords: current density-voltage, organic solar cell, quasi-linear monotonic increase

Posted Date: June 4th, 2021

DOI: https://doi.org/10.21203/rs.3.rs-566375/v1

License: (c) (i) This work is licensed under a Creative Commons Attribution 4.0 International License.

Read Full License

Version of Record: A version of this preprint was published at Scientific Reports on August 18th, 2021. See the published version at https://doi.org/10.1038/s41598-021-96222-w. 


\section{Abstract}

We investigated the variation of current density-voltage $(\mathrm{J}-\mathrm{V})$ characteristics of an organic solar cell (OSC) in the dark and at 9 different light intensities ranging from 0.01 to 1 sun of the AM1.5G spectrum. All three conventional parameters, short-circuit currents $\left(J_{\mathrm{sc}}\right)$, open-circuit voltage $\left(V_{\mathrm{oc}}\right)$, and Fill factor (FF), representing OSC performance evolved systematically in response to light intensity increase. Unlike $J_{\mathrm{sc}}$ that showed quasi-linear monotonic increase, $V_{\mathrm{oc}}$ and FF showed distinctive non-monotonic variations. To elucidate the origin of such variations, we performed extensive simulation studies including Shockley-Read-Hall (SRH) recombination losses. Simulation results were sensitive to defect densities, and simultaneous agreement to 10 measured $J-V$ curves was possible only with the defect density of 5 * $10^{12} \mathrm{~cm}^{-3}$. Based on analyses of simulation results, we were able to separate current losses into SRHand radiative-recombination components and, moreover, identify that the competition between $\mathrm{SRH}$ - and radiative-loss currents were responsible for the aforementioned variations in $J_{\mathrm{sc}}, V_{\mathrm{oc}}$, and FF. In particular, we verified that apparent demarcation in $V_{\mathrm{OC}}$, and FF variations, which seemed to appear at different light intensities, originated from the same mechanism of dominance switching between recombination losses.

\section{Full-text}

Due to technical limitations, full-text HTML conversion of this manuscript could not be completed. However, the manuscript can be downloaded and accessed as a PDF.

\section{Figures}




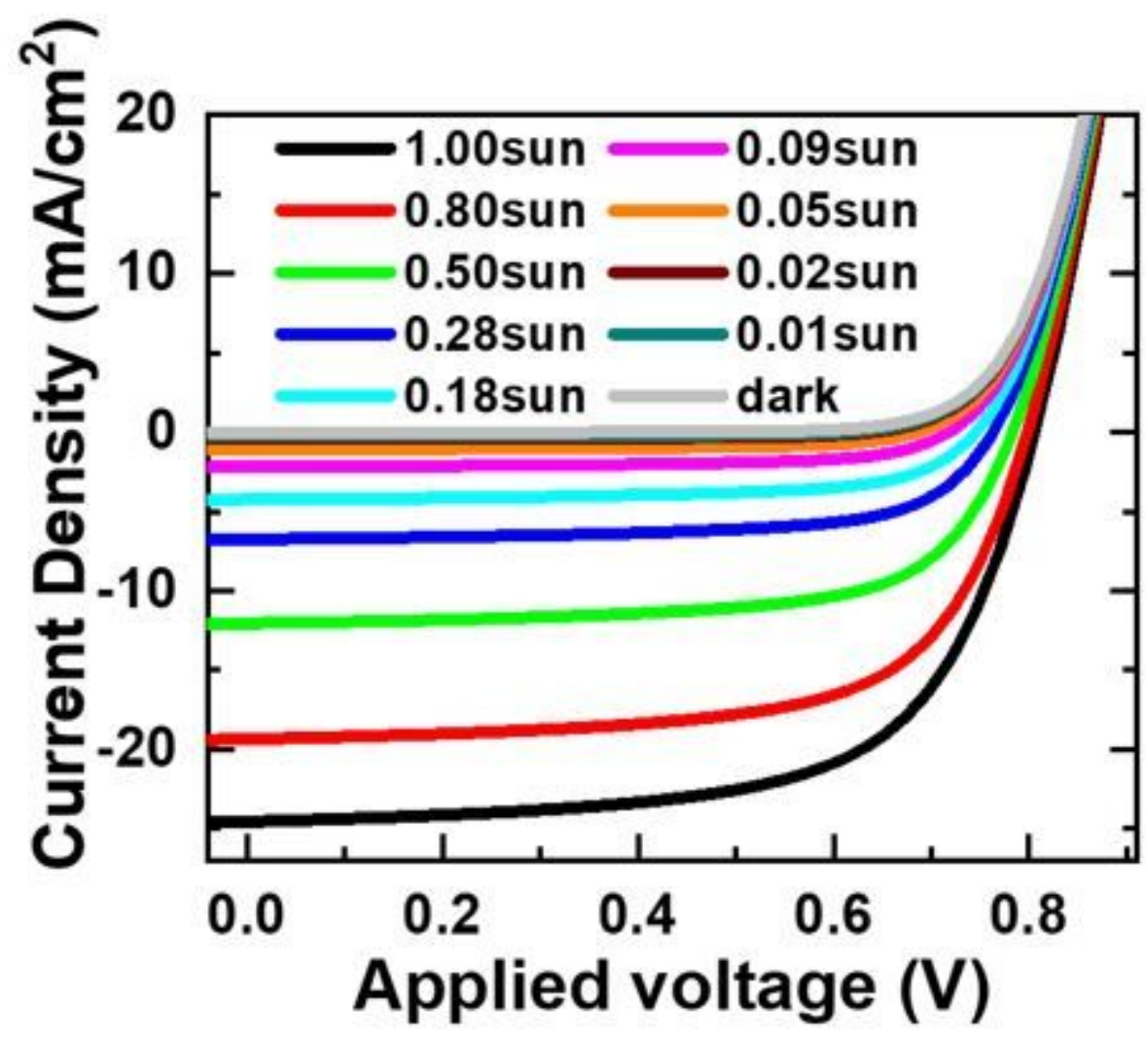

Figure 1

Light intensity dependence of $\mathrm{J}-\mathrm{V}$ characteristics of the OPV. 

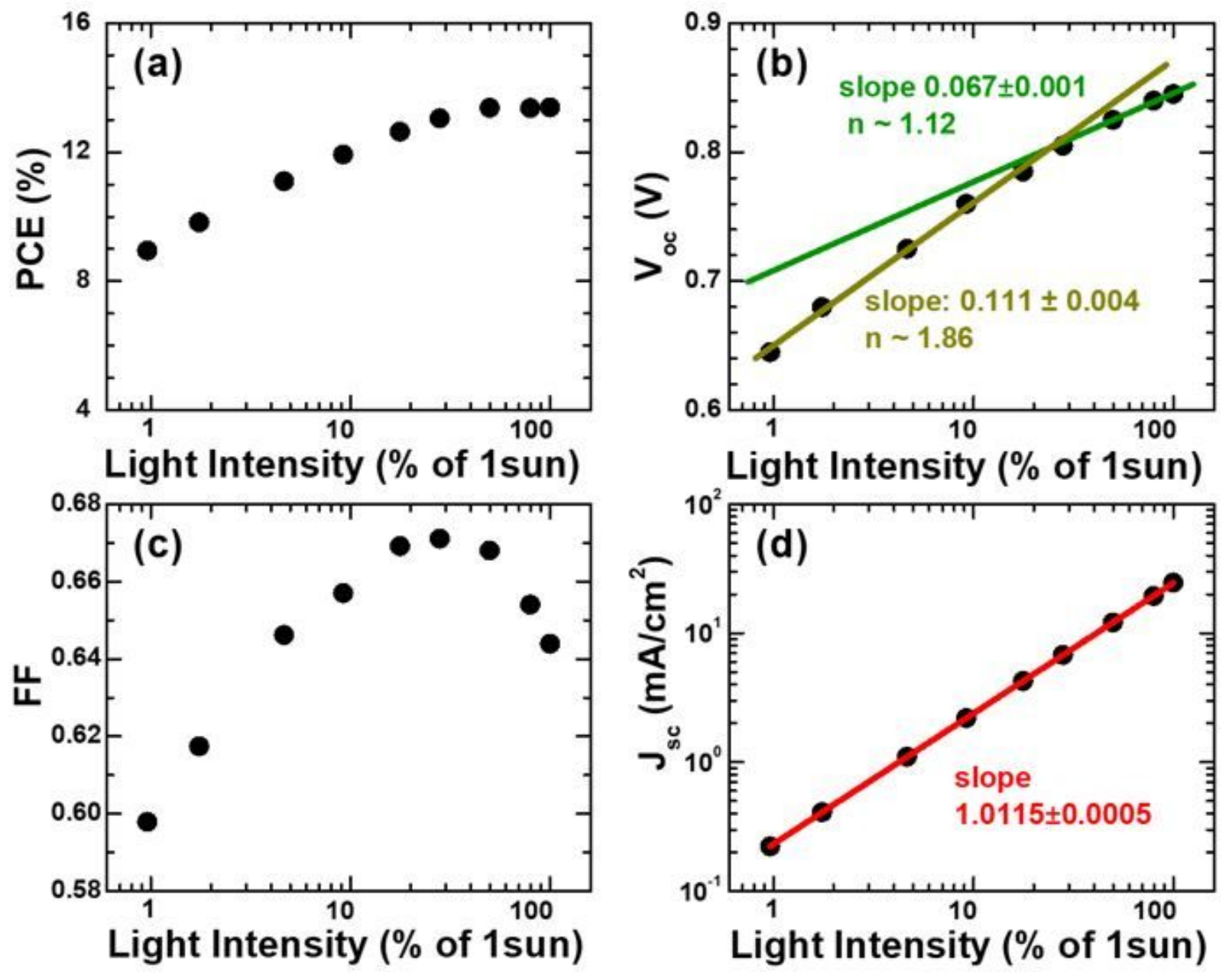

Figure 2

Light-intensity dependent solar cell parameters: (a) power conversion efficiency PCE, (b) open-circuit voltage Voc, (c) fill factor FF, and (d) short-circuit current density Jsc. 

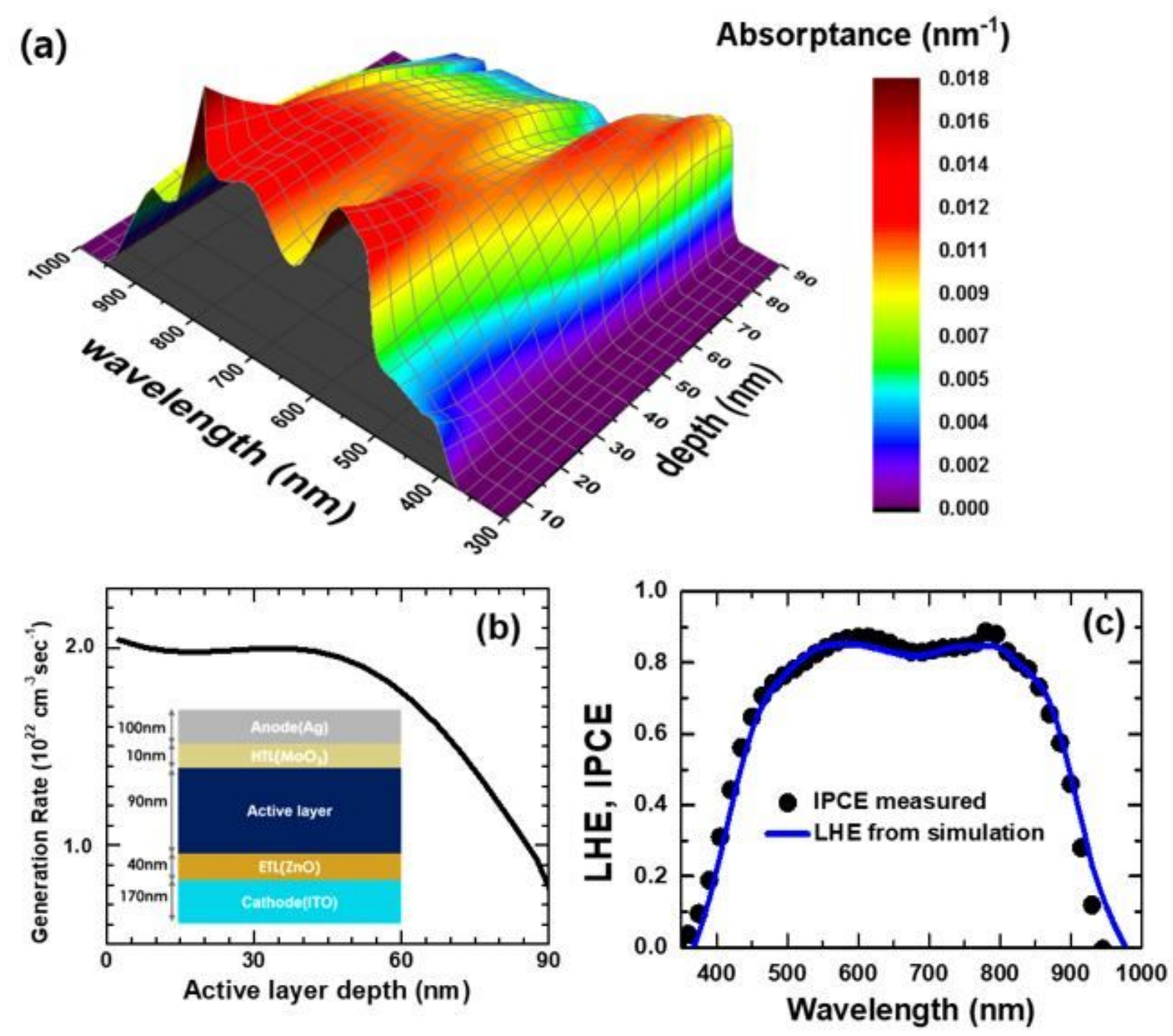

Figure 3

Optical simulation results: (a) position-dependent absorptance spectra within an active layer, (b) depth profile of exciton generation rates and a multilayer OSC structure (inset), and (c) the comparison of spectra of light-harvesting efficiency and measured incident photon-to-electron conversion efficiency. 

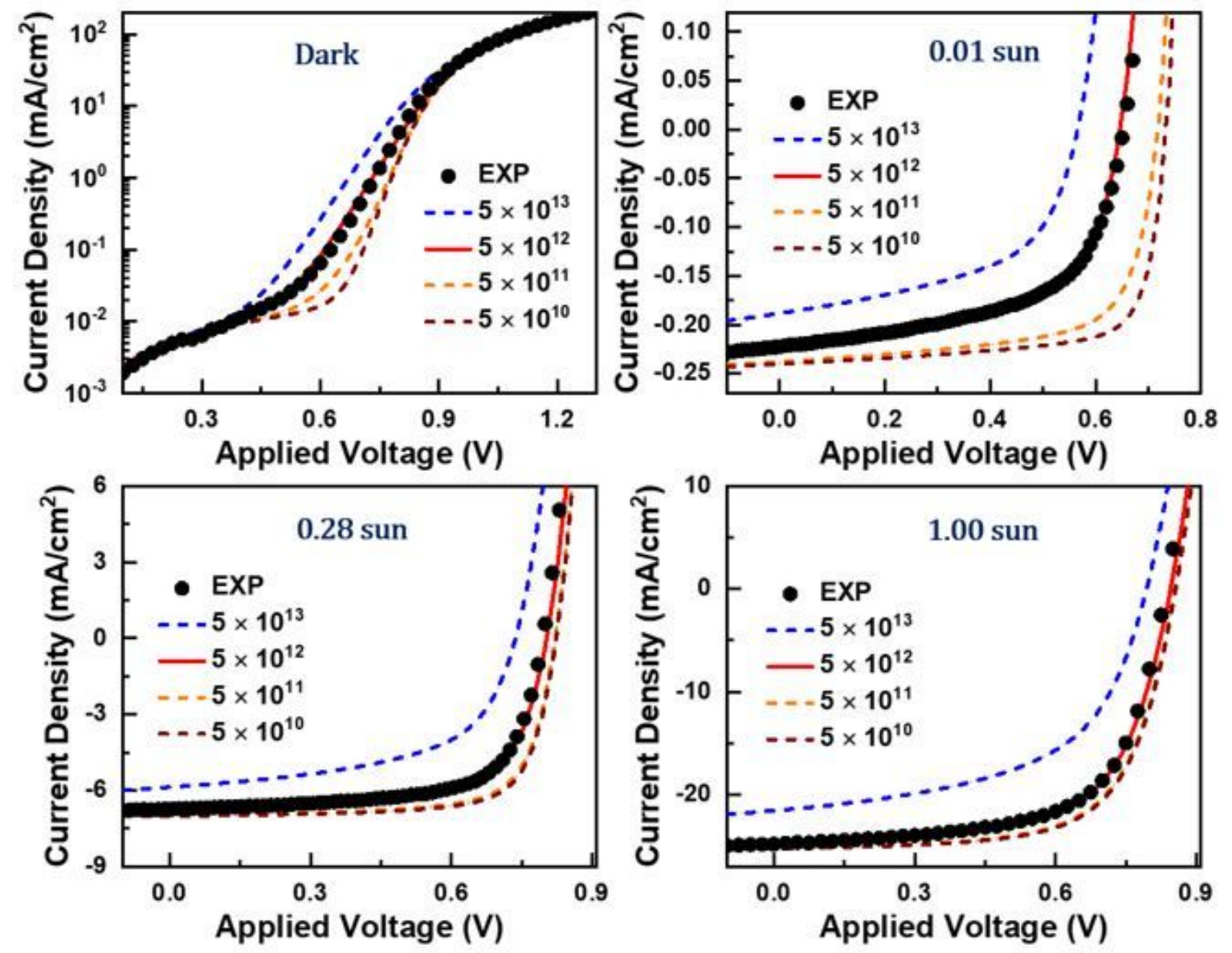

Figure 4

Comparison of simulated and measured J-V curves. Four orders of defect density variation were assumed for a set of simulated J-V curves. Simulations agreement of simulation results with experimental J-V curves measured in the dark, and at weak ( 0.01 sun), medium ( 0.28 sun), and strong (1 sun) illumination conditions appear only with defect density of . 

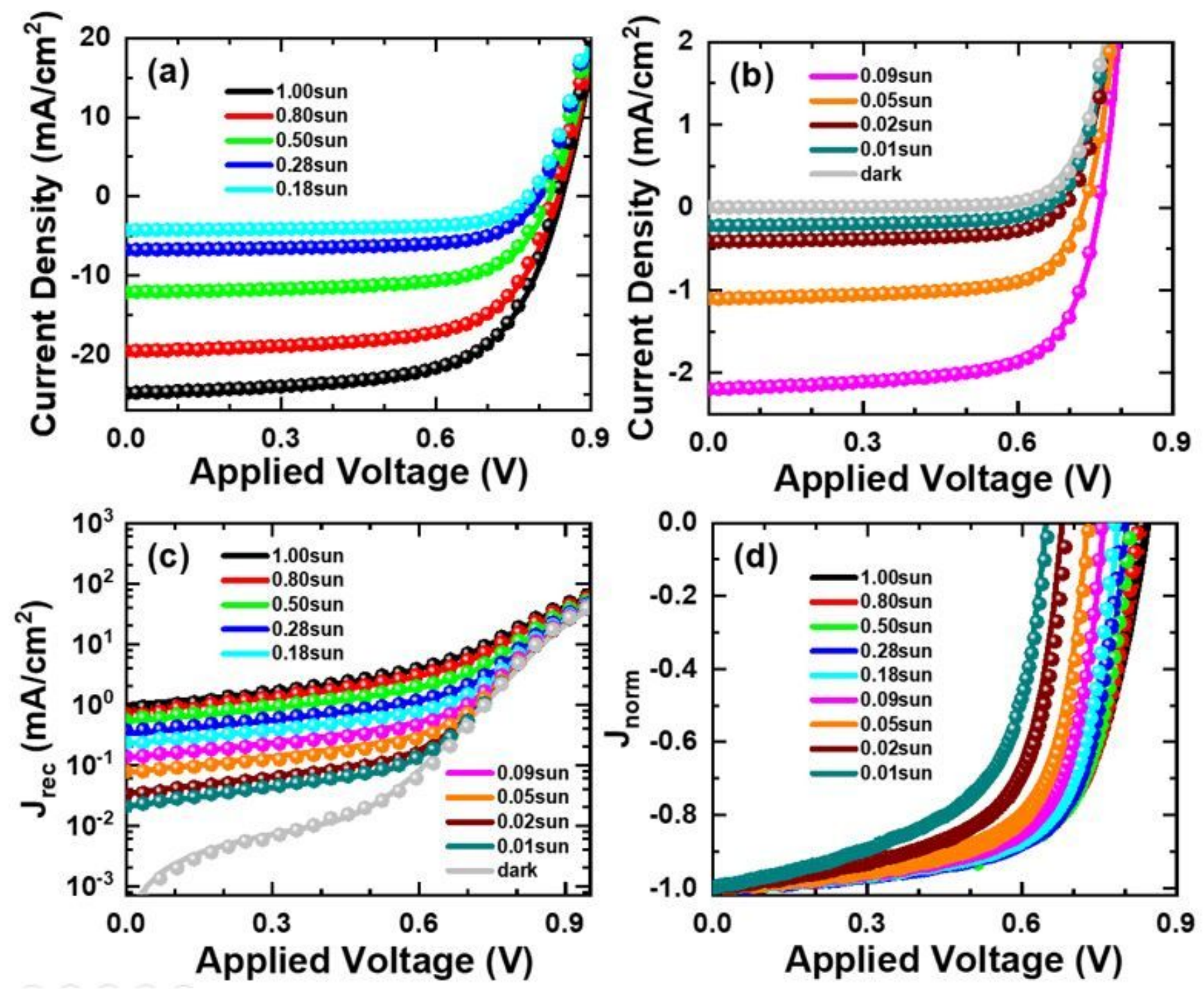

Figure 5

Comparison of simulated J-V curves (solid lines) with the corresponding data (symbols) measured at 9 different light intensities and also in the dark: (a) from 0.18 to 1.00 sun, and (b) below 0.09 sun and in the dark. (c) Comparison of simulated recombination current curves, Jrec-V (solid lines), with the sum (symbols) of measured $\mathrm{J}(\mathrm{V})$ and simulated generation current Jgen $(\mathrm{L})$ at each light intensity $\mathrm{L}$. (d) Variations of normalized current densities Jnorm with respect to applied bias voltages: . 

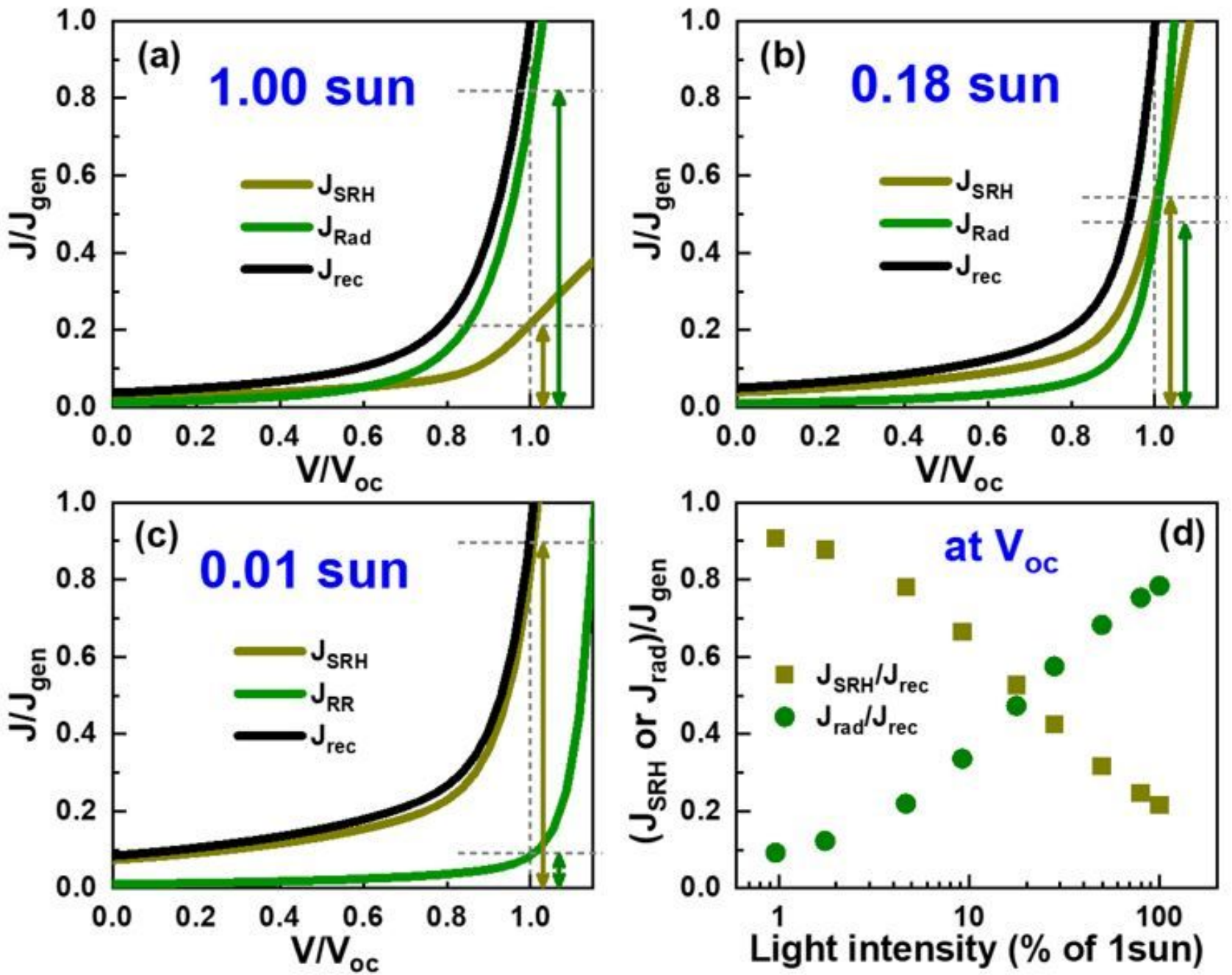

Figure 6

Variations of simulated $\mathrm{SRH}$, radiative, and total recombination currents with respect to applied voltages correspond to (a) weak (0.01 sun), (b) medium ( 0.18 sun), and (c) strong (1 sun) illumination conditions. Recombination currents are normalized by generation currents $\mathrm{Jgen}(\mathrm{L})$, and bias voltages are normalized by Voc. (d) Variations of SRH- and radiative-recombination currents at Voc with respect to light intensities. 

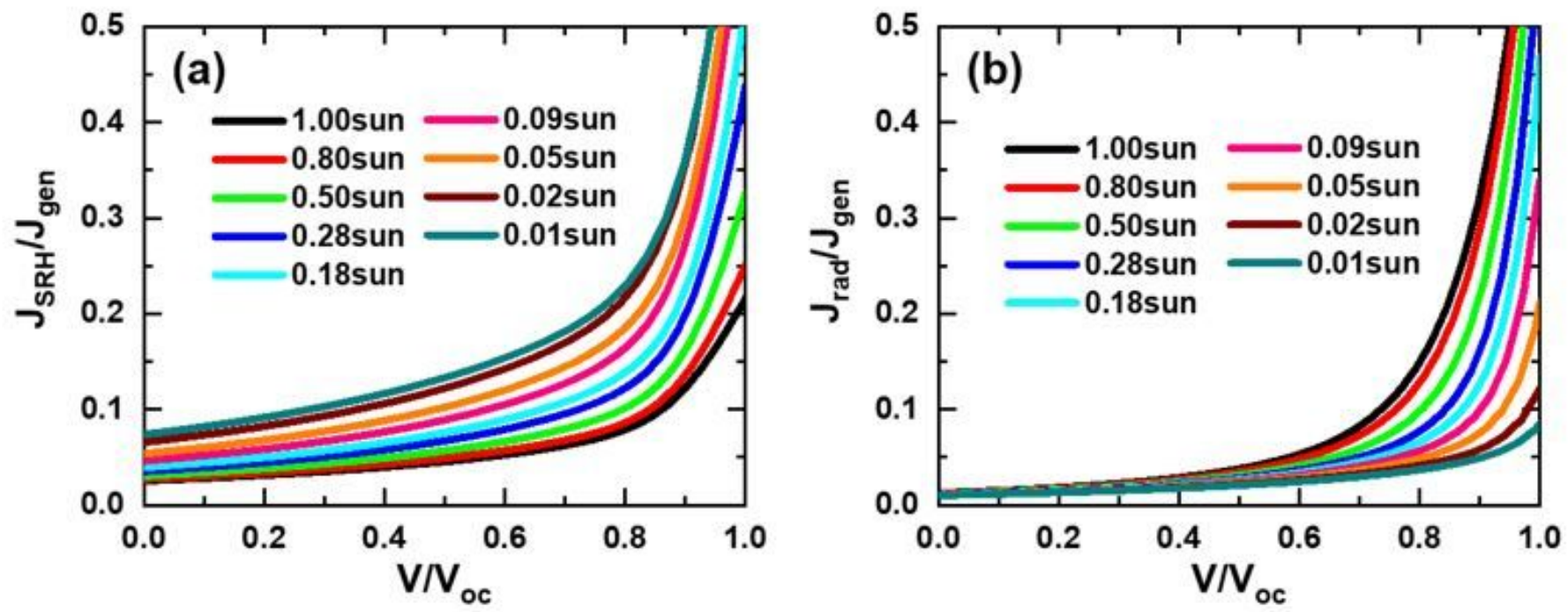

Figure 7

Light-intensity dependent variations of normalized $\mathrm{SRH}$ - and radiative-recombination currents with respect to normalized applied bias voltages: (a) JSRH/Jgen versus V/Voc and (b) Jrad/Jgen versus V/Voc. 

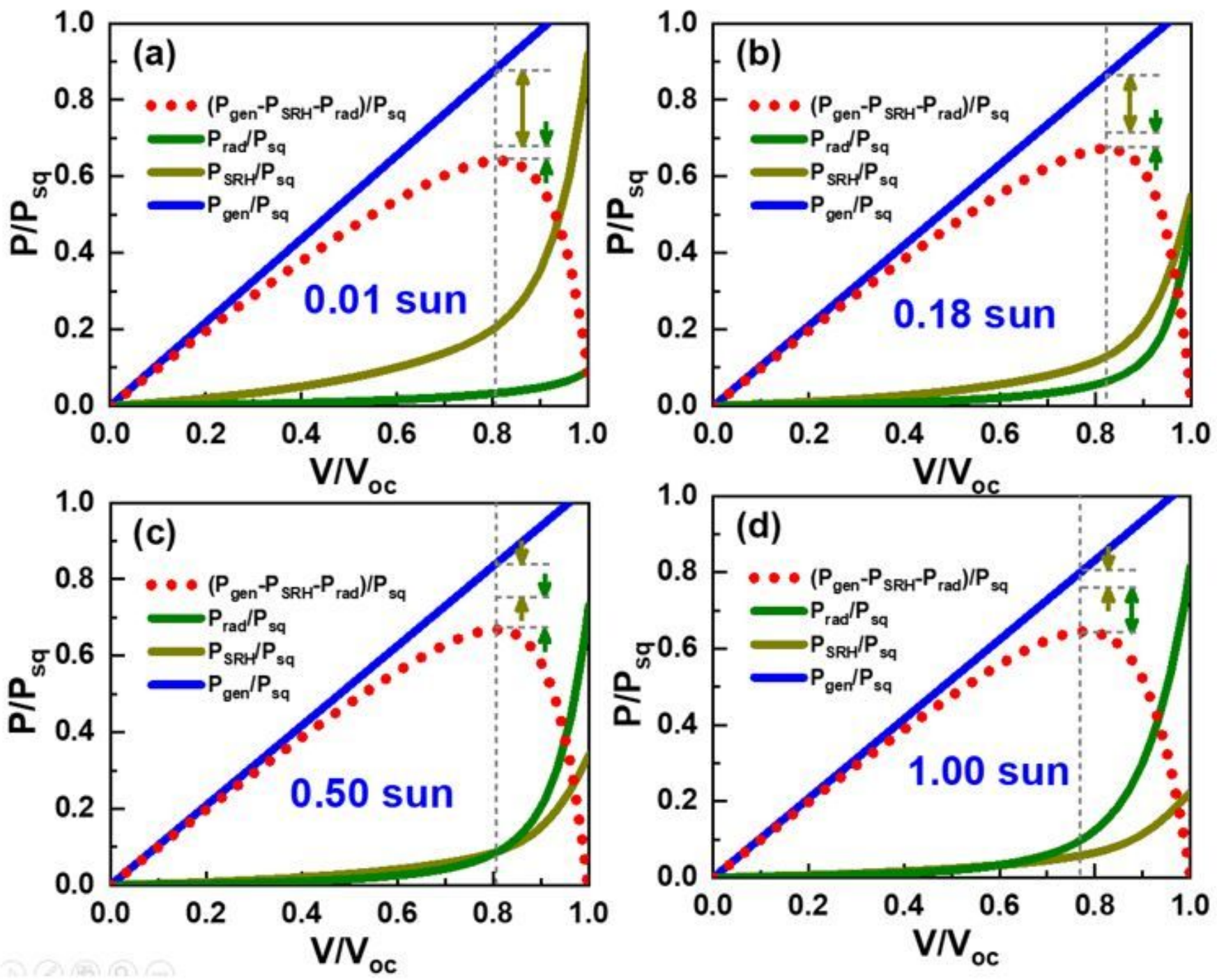

Figure 8

Variations of normalized power components with respect to normalized applied bias voltages corresponding to light intensities of (a) 0.01, (b) 0.18, (c) 0.50, and (d) 1.00 sun. Each power component is normalized by Psq that is defined as the product of Jsc and Voc, and applied bias voltages are normalized by Voc. 

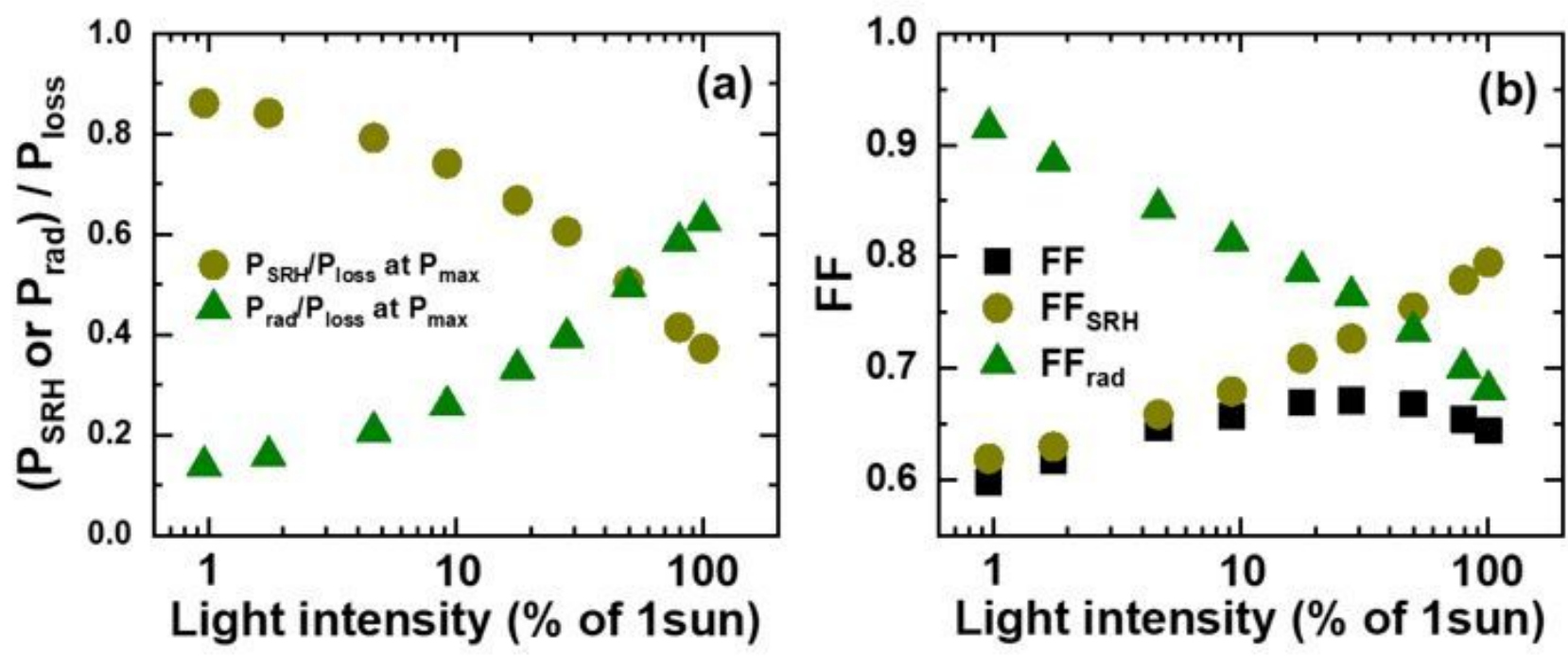

Figure 9

(a) Variations of the relative contributions of power losses PSRH and Prad to the total power loss Ploss at the maximum power points with respect to light intensities. (b) Variations of fill factors (FFs) with respect to light intensities. FFSRH and FFrad are FFs that would appear if only PSRH or Prad were responsible for power loss.

\section{Supplementary Files}

This is a list of supplementary files associated with this preprint. Click to download.

- supportinginformationScientificReportssubmitted.docx 